\title{
Réseau intercontinental d'approvisionnement énergétique - scénario pour un tracé de la ligne de transport électrique de l'Afrique du Nord à l'Europe centrale
}

Intercontinental network of energy supply - Scenario for a power line route from North Africa to Central Europe

Interkontinentale Vernetzung der Energieversorgung - Szenario für eine

Stromleitungstrasse von Nordafrika nach Mitteleuropa

Katrin Walter and Stephan Bosch

\section{(2) OpenEdition}

\section{Electronic version}

URL: http://journals.openedition.org/rge/5447

ISSN: 2108-6478

\section{Publisher}

Association des géographes de l'Est

Printed version

Date of publication: 1 January 2015

ISSN: 0035-3213

\section{Electronic reference}

Katrin Walter and Stephan Bosch, « Réseau intercontinental d'approvisionnement énergétique scénario pour un tracé de la ligne de transport électrique de l'Afrique du Nord à l'Europe centrale ", Revue Géographique de l'Est [Online], vol. 55 / n¹-2 | 2015, Online since 09 June 2015, connection on 08 September 2020. URL : http://journals.openedition.org/rge/5447 


\section{Réseau intercontinental}

d'approvisionnement énergétique scénario pour un tracé de la ligne de transport électrique de l'Afrique du Nord à l'Europe centrale

Intercontinental network of energy supply - Scenario for a power line route from North Africa to Central Europe

Interkontinentale Vernetzung der Energieversorgung - Szenario für eine

Stromleitungstrasse von Nordafrika nach Mitteleuropa

Katrin Walter and Stephan Bosch

\section{Problématique et objectifs}

1 Les changements en cours dans les pays d'Afrique du Nord marqués par des processus de démocratisation pourraient former la base d'un approvisionnement énergétique sûr basé sur l'énergie solaire et faciliter la coopération avec les voisins européens. C'est du moins l'espoir des protagonistes de l'ambitieux concept Desertec, dont la version présentée d'un flux de courant renouvelable venant du désert est considérée comme étant d'un format de style néo-colonial. Ce soupçon a été alimenté par le fait que seulement une des 13 sociétés fondatrices de l'initiative de l'industrie Dii GmbH est issue d'un pays potentiellement producteur (en l'occurence la société Cevital en Algérie). Le reste est représenté par les grandes entreprises principalement allemandes comme E.ON, RWE, Schott Solar et Siemens, dont l'intérêt pour l'Afrique du Nord est principalement le résultat d'un calcul de politique industrielle (Schmitt 2012, p 257). À ce point de vue cependant, on peut rétorquer, que selon la conception prévue ce sont les pays producteurs qui doivent bénéficier de la plus grande partie de la valeur 
ajoutée, notamment en lien avec le développement des industries locales et le transfert de connaissances techniques (Fondation Desertec 2009; DLR 2009a; Löfken 2011a). En outre, la majorité $(80 \%)$ de l'électricité solaire produite restera dans les pays de la région (MENA ) et seulement une partie de celui-ci servira à couvrir la demande d'électricité européenne. Des États comme le Maroc réalisent déjà leur propre plan solaire, indépendamment de Desertec et pensent aussi à l'exportation d'électricité à (Löfken 2011b, p.27). On peut cependant se demander comment et surtout le long de quels corridors sera transportée cette part de la production de courant d'Afrique du Nord vers les centres éloignés de consommation en Europe centrale. Cela nécessite la création d'un système efficace de transport de courant à très haute tension (HVDC). Avec cette technologie on ne perd que 2 à $4 \%$ de l'énergie par millier de kilomètres. Cela permettrait d'approvisionner, de manière rentable, avec de l'électricité renouvelable des centres de consommation situés à une distance de 3000 à $5000 \mathrm{~km}$.

Dans la présente étude il s'agit de comprendre les facteurs spatiaux influençant la conception du tracés des lignes haute tension et de les représenter en utilisant des SIG. À cet effet, dans une première étape, on a identifié les centres de consommation actuels en Europe, qui constituent les extrémités des lignes de transport d'électricité. De plus, de vastes zones de restriction pour le passage des lignes électriques sont projetées dans la zone d'étude, si bien qu'en principe émergent des corridors spatiaux utilisables. Enfin, compte tenu de facteurs de coûts fixes spécifiques, on a calculé et visualisé le tracé optimal d'un corridor de transit du courant électrique entre le site de la centrale de Ain Beni Mathar et le centre économique de forte consommation qu'est Munich. Par rapport à l'étude de la DLR (2009), le présent article propose une résolution plus élevée et donc une analyse spatiale plus détaillée. De plus, on discute des progrès technologiques et des caractéristiques des sites, y compris la bathymétrie ainsi que de la question des distances acceptables.

\section{Les fondements techniques}

3 Dans le cadre de la coopération trans-méditerranéenne des énergies renouvelables (TREC) (un réseau de politiciens, de scientifiques et d'économistes) le concept DESERTEC a été développé à l'initiative du Club de Rome dans les années 2003-2007. En 2009, il a été repris par le réassureur Munich Re ainsi que douze autres sociétés. Le but est de produire de l'électricité renouvelable dans les zones où le rayonnement solaire direct est élevé et donc d'approvisionner à la fois les pays producteurs et les centres de consommation éloignés. Selon les différents potentiels nationaux au sein de l'EUMENA (Europe + la région MENA) toutes les formes d'énergies renouvelables doivent à long terme être incluses et reliées par un réseau électrique intercontinental (Dannenberg et al. 2012, p 40). Jusqu'à présent, l'accent est cependant mis sur l'utilisation de l'énergie solaire. C'est notamment en raison du grand potentiel que représentent les zones désertiques de la terre qui, en l'espace de six heures, reçoivent plus d'énergie due au rayonnement solaire que la consommation de l'ensemble de l'humanité au cours d'une année. Avec environ 3000 heures de soleil par an, cela représente un rendement d'environ 2.500 kWh par mètre carré (Schmitt 2012, p 244; Wandler 2011, p 94). Ce potentiel peut être exploité efficacement par les technologies solaires thermiques (CSP) comme les alignements de miroirs paraboliques et les collecteurs de Fresnel, ainsi que les tours solaires et les systèmes Dish-Stirling (cf. Heuer 2011; Kaltschmitt et al. 2006; 
Quaschning 2011). L'avantage de ces solutions technologiques est que par couplage avec des systèmes de stockage de chaleur (par exemple par sel fondu, béton.) on peut les utiliser comme source d'énergie de charge de base (Schmitt 2012, p 246; Bosch 2010, p 28 et suivantes).

Le problème est que l'énergie solaire à concentration thermique ne pourra atteindre la compétitivité avec l'électricité produite conventionnellement qu'en 2030 (Rentzing 2011, p 50). Il faut noter par ailleurs, que la pression concurrentielle de la part du photovoltaïque ne cesse d'augmenter. Ceci est principalement dû au marché beaucoup plus mûr de l'énergie photovoltaïque, car en raison d'une capacité installée mondiale de $70 \mathrm{GW}$ d'ici la fin de 2011, l'industrie a réussi à réaliser des économies d'échelle, si bien qu'elle génère des coûts de production à moins de 10 cents / $\mathrm{kWh}$ dans les endroits ensoleillés $(2500 \mathrm{kWh} / \mathrm{m} 2 / \mathrm{a})$. En revanche, la capacité totale mondiale de solaire à concentration thermique s'élève à seulement deux gigawatts, soit trop peu pour rester compétitif avec la production de masse et la standardisation. Le coût final des technologies CSP sont encore entre 18 et $24 \mathrm{ct} / \mathrm{kWh}$. Par conséquent, les centrales cylindro-paraboliques et les tours solaires ont perdu leur attractivité initiale (voir Lessner 2010) dans les régions désertiques du monde (voir Heup 2012).

5 L'avantage significatif de coût de l'énergie photovoltaïque se manifeste maintenant par le fait que des entreprises comme Solar Millennium, qui ont été fortement impliquées dans le marché de la CSP ont fait faillite. De nombreux projets qui ont été fondés à l'origine sur la technologie de la CSP, se reconvertissent maintenant dans les cellules photovoltaïques plus lucratives. Dans ce contexte, il est compréhensible que le géant industriel Siemens se soit retiré de l'activité de la CSP autrefois prometteuse. Finalement, l'avantage des centrales thermiques solaires repose uniquement sur la possibilité de fournir une alimentation en énergie de charge de base au moyen de stockage. Même les rendements élevés des technologies CSP, ne sont plus un avantage particulier avec le développement de cellules à concentration photovoltaïque (CPV) ayant des rendemnts supérieurs à $40 \%$. Le triomphe de l'énergie photovoltaïque est donc accélérée par la technologie CPV. Même l'Agence marocaine pour l'énergie solaire (Masen: Moroccan Agency for Solar Energy), qui a promu principalement l'expansion des technologies CSP, a montré un grand intérêt pour le photovoltaïque de concentration puisqu'au début de l'année 2012 elle a mis en service à Ouarzazate la première centrale d'une capacité de $160 \mathrm{MW}$ (Schmitt 2012, p 258). Seuls les États qui font un calcul de politique industrielle, c'est à dire qu'ils comptent principalement sur la valeur ajoutée dans leur propre pays et non sur les coûts de production, sont toujours intéressés à l'expansion des centrales thermiques solaires. Les espoirs reposent sur l'Arabie saoudite, parce qu'une expansion de $20 \mathrm{GW}$ de la capacité de concentration solaire thermique y est envisagée (voir Schwarzburger 2012a et 2012b). L'énergie propre ainsi produite doit être consommée seulement sur place afin de se substituer aux combustibles fossiles réservés pour des exportations lucratives. Dans le contexte de cette stratégie, il est aussi compréhensible que l'Arabie saoudite cherche aussi à développer des centrales nucléaires. Dans tous les cas, l'intégration du plus grand exportateur de pétrole au monde dans un réseau intercontinental d'exportation de courant continu n'est pas pertinente (Löfken 2011, p 28). 


\section{Le transport du courant continu}

6 La mise en œuvre du projet de production de courant dans les déserts, soutenu par des sociétés financièrement solides tels que RWE, E.ON, Schott Solar, Flagsol, Abengoa Solar, la Banque allemande et HSH Nordbank, se concentre actuellement sur la région EUMENA, à savoir l'Europe, le Proche-Orient et l'Afrique du Nord (voir Fondation Desertec en 2009, Trieb 2012). Les premières centrales solaires thermiques ont déjà été créées En Afrique du Nord, même si ces projets ne sont pas toujours associés à Desertec, mais doivent être compris comme des initiatives indépendantes des différents Etats. Le développement de ces projets est fortement encouragé dans un État tributaire des importations d'énergie fossile comme le Maroc (la dépendance énergétique est actuellement de 97\%) et prend forme concrètement avec les centrales thermiques solaires de Ain Beni Mathar et de Ouarzazate (voir Bine Service d'information 2011; Wandler 2011, p 92). La Tunisie veut aussi utiliser son grand potentiel solaire et pour cela prévoit de construire la tour solaire de TuNur en 2014 (Schwarzburger 2012b, p 57). À partir de 2016 TuNur doit produire de l'électricité pour l'Italie située au nord; mais le tracé exact du corridor de transport de l'électricité n'est pas encore fixé. Jusqu'à présent, on sait seulement que le corridor ne devrait pas passer par la Sicile, mais en mer entre la Sicile et la Sardaigne en direction de Rome (voir TuNur 2012).

Indépendamment du fait que le potentiel énergétique de l'Afrique du Nord soit exploité en utilisant le solaire thermique ou photovoltaïque, et quel que soit le cadre étatique ou transnational dans lequel ces technologies seront développées, le but est qu'au moins une partie de l'électricité produite soitv transportée en Europe. Cela nécessite l'utilisation de lignes de transport HVDC qui sont à installer à la fois en mer et sur terre. Avec ces lignes on peut transporter l'énergie sous forme de courant continu avec des tensions allant jusqu'à $150 \mathrm{kV}$ pour une capacité de $500 \mathrm{MW}$ et des pertes relativement faibles sur de longues distances. Il est prévu que d'ici 2020, des lignes à courant continu avec une tension de $500 \mathrm{kV}$ et une capacité de $2000 \mathrm{MW}$ deviennent la norme. Il existe déjà des lignes électriques puissantes, comme la ligne HVDC NorNed qui a été achevée en 2008 (un câble sous-marin entre la Norvège et les Pays-Bas, d'une longueur de $580 \mathrm{~km}$ qui est le plus long du monde (Zimmermann/ Weinhold 2011, p 23 $A B B$ AB 2008). En 2011, les travaux de construction de BritNed, une ligne de transmission HVDC entre la Grande-Bretagne et les Pays-Bas, ont été achevés ( $A B B A B$ 2008; Zimmermann/ Weinhold 2011, p 23). La construction de lignes électriques à haute performance entre l'Afrique du Nord et l'Europe est un terrain scientifique et technique nouveau, à l'exception de deux études de la DLR (2006 u. 2009b) et nécessite donc des travaux préparatoires à base de SIG. Divers facteurs spatiaux de doivent être inclus dans la planification. Le transfert de courant par les lignes CCHT a des impacts importants dans le paysage et est donc exclue pour des espaces sensibles. L'utilisation de SIG permet de synthétiser les caractéristiques paysagères existantes et de déterminer le cas échéant, les itinéraires favorables ou inappropriées. 


\section{Méthode}

\section{A. Les dimensions spatiales du transport de courant}

8 En plus de l'utilisation des corridors existants (par exemple des faisceaux de câbles le long des lignes de chemin de fer, qui peut être étendu à des lignes HVDC), l'analyse SIG est centrée sur l'identification de solutions spatiales alternatives. Pour avoir cette connaissance, il est d'abord nécessaire de déterminer les centres de consommation de l'énergie. Ensuite, il est nécessaire de définir les éléments d'exclusion pour la planification des lignes électriques, ainsi que de visualiser les zones de restriction correspondantes. Dans ce qui suit, on examine plus en détail les quatre facteurs principaux de localisation: les aires protégées sur terre et sur l'eau, l'utilisation des terres, les données bathymétriques (profondeur de la mer), ainsi que les valeurs de pente à partir des données orographiques.

\section{B. Les centres de consommation du courant}

9 Les centres de consommation actuels représentent les points d'aboutissement des lignes électriques, à savoir les sous-espaces qui ont une consommation nettement plus élevée. À partir du site de la centrale on peut calculer une connexion point-à-point. Les points cibles spécifiques sont définis par les coordonnées des transformateurs existants et potentiels qui convertissent le courant continu en courant alternatif. La présente analyse se concentre sur les centres de l'Union européenne (UE). À l'intérieur de l'UE, il existe des unités spatiales hiérarchisées; ce que l'on appelle la "Nomenclature des unités territoriales statistiques (NUTS)" (cf. UE 2003). Le niveau NUTS 0 signifie un État membre de l'UE, qui à son tour peut être subdivisé en trois niveaux supplémentaires. Il s'agit de NUTS 1 (3 à 7 millions d'habitants) pour les grandes régions (par exemple les Länder en Allemagne ou Zone d'études et d'aménagement du Territoire (ZEAT) en France), NUTS 2 ( 0,8 million à 3.000 .000 d'habitants) pour les régions de taille moyenne (par exemple districts administratifs en Allemagne ou régions en France) et NUTS 3 $(0,15$ à 0,8 million d'habitants) pour les régions plus petites (par exemple. Landkreise ou villes en Allemagne, ou départements en France) (voir figure Europa 2008).

Pour identifier les centres de consommation, il est nécessaire de prendre en compte la consommation totale d'énergie à une échelle spatiale la plus petite possible. Par un système de pondération on peut déduire les valeurs de consommation pour le niveau NUTS 3 des valeurs disponibles pour NUTS 0 . À cet effet, la consommation d'énergie d'un pays de l'UE (NUTS de niveau 0) est d'abord répartie entre les services, le secteur domestique et le secteur industriel. La consommation d'énergie au niveau NUTS 3 est calculée à partir de la valeur des PNB pour les secteurs des services et de l'industrie, tandis que pour la consommation domestique on utilise l'effectif de la population (Leuthold et al., 2008, p 18 f.). On suppose que le rapport entre la valeur ajoutée brute dans une unité NUTS 3 et la valeur ajoutée brute totale (NUTS 0 unité) correspond au rapport de la consommation d'énergie dans une unité NUTS 3 avec la consommation totale de courant (NUTS 0). Pour cette raison, la part de la valeur ajoutée brute du secteur industriel d'une unité NUTS 3 dans la valeur industrielle ajoutée brute de l'ensemble de l'Etat est d'abord évaluée. Cette valeur est ensuite multipliée par la consommation industrielle totale d'électricité de chaque État membre. Il en résulte la 
consommation d'électricité du secteur industriel dans une unité NUTS 3. On procède de la même façon pour le secteur des services. Afin de déterminer la consommation d'énergie des ménages, on calcule la part de la population de l'unité NUTS 3 dans la population totale et on le multiplie par le courant total consommé par le secteur domestique. Les valeurs pour les trois secteurs sont ensuite ajoutées, de sorte que l'on dispose de la consommation exacte de courant pour chaque unité NUTS 3. Enfin, afin de comparer les unités NUTS de tous les pays, les résultats sont encore normalisés par kilomètres carrés.

\section{Les espaces d'exclusion}

\section{a. Les espaces protégés}

11 Les impacts paysagers qui pourraient être produits par les lignes CCHT ne sont pas autorisés dans de nombreuses zones protégées. Par conséquent, il est nécessaire d'exclure les zones environnementales sensibles du passage des lignes. Les données des zones Natura 2000, un ensemble de données sur les aires protégées de l'Union européenne dans le cadre des réserves de la directive Faune-Flore-Habitat (FFH) et de la directive Oiseaux (voir 2011a pour l'EEE) sont d'une importance particulière pour l'analyse SIG. À celà s'ajoutent les données de la base mondiale sur les aires protégées, un projet conjoint du Programme des Nations Unies pour l'environnement (PNUE) et de l'Union Internationale pour la Conservation de la Nature (UICN). Celles-ci rassemblent les zones protégées rurales et marines. Dans une autre étape, il est nécessaire d'examiner dans quelle mesure ces zones protégées peuvent permettre la construction de lignes. Les données de l'UICN qui comprennent sept catégories de protection internationale ( $1 \mathrm{a}, 1 \mathrm{~b}, 2,3,4,5$ et 6 ) (voir UICN 2012) sont fondamentales. Les deux dernières catégories (5 et 6) par exemple correspondent à la la nécessité de déplacer le tracé des lignes. La catégorie 5 est classée comme type d'espace "paysage protégé / aire marine protégée" et la catégorie 6 correspond au type d'espace "gestion de la zone de protection des ressources" (voir WWF Allemagne 2008). La plupart des parcs naturels régionaux et des zones protégées ressortent comme des espaces à protéger des tracés des lignes. En tout état de cause, les parcs nationaux, les réserves naturelles et les «zones protégées» sont à exclure, par exemple le sud-ouest du Jura souabe et la vallée du Danube, les massifs du Kaisergebirge et du Karwendel avec l'Isar (cf. figure 1). En raison de zones sensibles, il faut ajouter une distance supplémentaire de $300 \mathrm{~m}$ à la zone d'exclusion pour la pose des lignes. Ces zones tampons doivent permettre d'éviter que les lignes soient implantées directement en bordure des limites des zones protégées et puissent ainsi nuire à l'acceptabilité par la population. Cependant, il n'existe pas de législation uniforme concernant les distances, car jusqu'à présent on n'a guère d'expérience concernant l'acceptabilité des lignes électriques. 


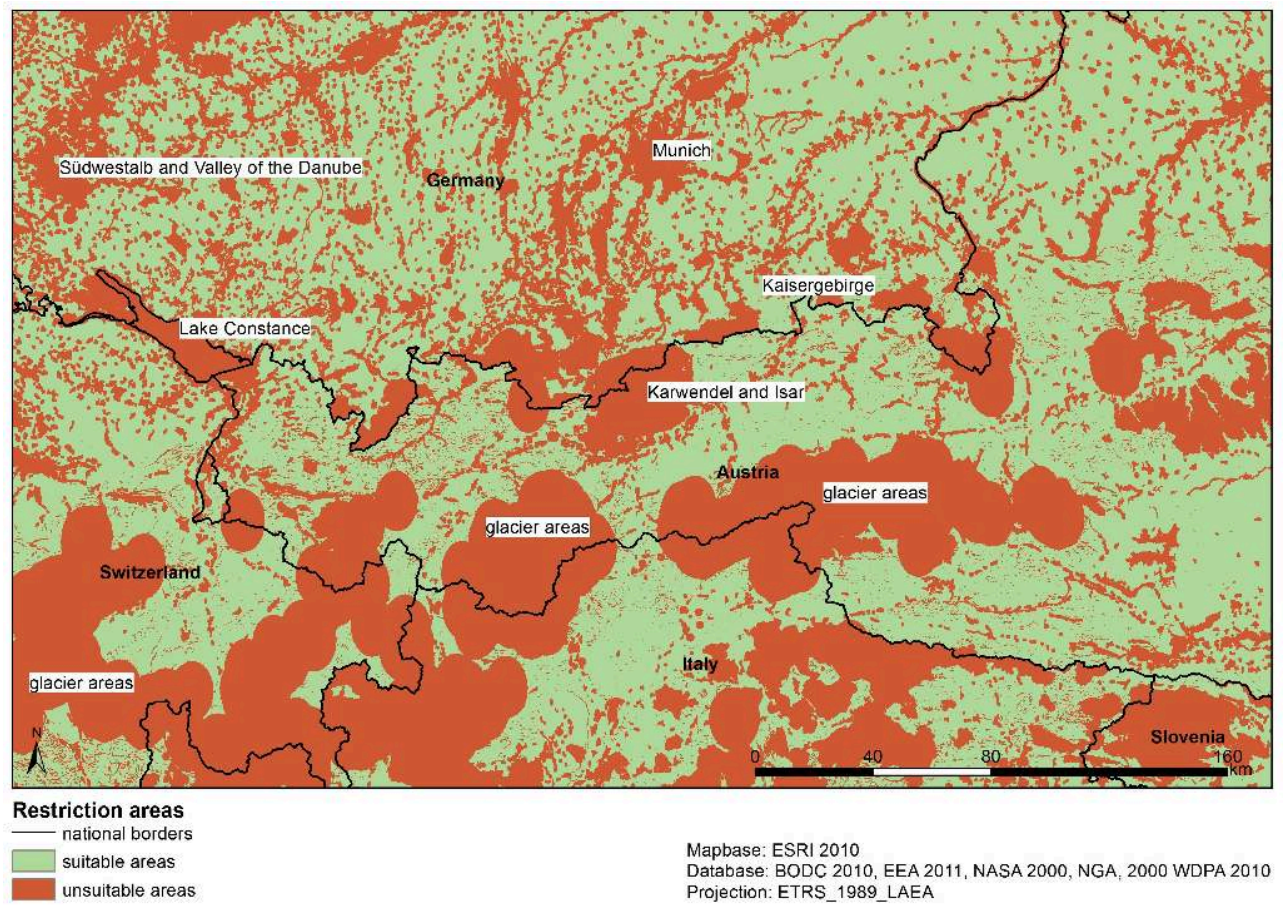

\section{b. L'utilisation des sols}

Pour pouvoir détecter les structures de surface du paysage, les données d'utilisation des terres sont incluses dans l'analyse SIG. Pour cela, on se sert de la base de données Corine Land Cover de l'Agence européenne pour l'environnement sert (voir EEA 2011b). Ces données de couverture du sol comprennent 44 classes d'utilisation des terres, qui appartiennent à cinq grandes catégories - «environnement bâti», «terres agricoles», «forêts et espaces naturels», «zones humides» et «surfaces d'eau» - (voir DLR 2012). De la même façon, il s'agit aussi d'identifier les domaines qui ne se prêtent pas à l'installation de lignes HVDC. Comme il n'existe pas de réglementation uniforme au sein de l'UE, la réglementation de l'aménagement du territoire de la République fédérale d'Allemagne peut être en partie transférée à l'UE. Par exemple les zones de la catégorie "tissu urbain continu" (voir la figure DLR 2012), les zones glaciaires dans les Alpes ainsi que les plans d'eau (voir Fig. 1) doivent être exclues. Les zones de restriction sont aussi élargies par des distances supplémentaires (par exemple de $400 \mathrm{~m}$ pour le tissu urbain, de $100 \mathrm{~m}$ pour les zones industrielles et commerciales, de $2.000 \mathrm{~m}$ pour les aéroports et de $100 \mathrm{~m}$ pour les plans d'eau), de sorte qu'il constitue le second masque de la zone d'exclusion.

\section{c. La bathymétrie comme critère d'exclusion}

Pour transporter l'électricité renouvelable de l'Afrique du Nord vers l'Europe, il est nécessaire de planifier la majorité du tracé des lignes électriques à travers la Méditerranée. Bien qu'une telle approche soit plus complexe et donc également associée à des coûts plus élevés, cela permet de contourner les pays politiquement instables comme l'Egypte, Israël, le Liban et la Syrie. Alors que ce n'est pas l'objet de la présente étude d'évaluer la stabilité politique des États-nations dans l'analyse SIG, il est 
clair qu'un grand nombre de pays de transit augmente le risque de pénurie d'approvisionnement. Cela ne signifie pas que les pays politiquement instables peuvent être entièrement contournés. Dans le cadre du concept énergétique trans-continental, ce n'est pas réaliste. De plus, les pays d'Afrique du Nord comme par exemple l'Algérie, appartiennent à l'OPEP et figurent ainsi depuis longtemps comme des partenaires énergétiques fiables. La mise en service du gazoduc Algérie Sardaigne Gasdotto Italia (GALSI) prévue pour 2014 peut être considérée comme la preuve de l'existence d'une association de coopération (voir GALSI 2011). De plus, on ne sait pas dans quelle mesure la France est prête à soutenir la construction de lignes électriques en provenance d'Espagne. Il faut aussi prendre en compte le fait que la société énergétique dominée par l'État Electricité de France (EDF), est principalement intéressée à pouvoir envoyer vers l'Europe centrale une partie de la production de ses 58 centrales nucléaires. La France redoute le courant éolien et solaire bon marché qui pourrait venir du Sud et conduirait à de plus grandes fluctuations sur le réseau électrique (Zimmermann/ Weinhold 2011, p 18).

14 Avec la technologie actuelle, il est possible de poser des lignes électriques à une profondeur marine allant jusqu'à $2000 \mathrm{~m}$. Il est probable cependant, que d'ici à la construction effective des lignes, la technologie sera suffisamment mûre pour pouvoir installer des lignes à des profondeurs encore plus importantes. Par conséquent, dans le cadre de l'analyse SIG, on peut exclure les parties de la Méditerranée avec des profondeurs inférieures à $3000 \mathrm{~m}$. Il est à noter cependant, qu'il y a relativement peu de fonds méditerranéens au-dessous de $3000 \mathrm{~m}$. Cependant, en raison de grandes surfaces contiguës d'exclusion, ces quelques zones sont peu perméables. D'une part, la zone sud de la mer Ionienne ainsi que des parties de la mer de Libye sont inadéquats pour la pose de câbles sous-marins. D'autre part, il existe des zones relativement petites d'exclusion dans la mer Tyrrhénienne et dans le sud de la Méditerranée. Aussi, la pose de câbles en Méditerranée occidentale est largement possible. En revanche, en Méditerranée orientale (au large des côtes de l'Egypte et de l'Est Libye), il y a de grandes barrières spatiales. Dans l'idéal, il faudrait poser un câble à travers le détroit de Gibraltar, parce qu'avec environ $14 \mathrm{~km}$, c'est la voie la plus courte entre les pays d'Afrique et le continent européen. Bien qu'un câble sous-marin ait déja été placé sur ce détroit, sa capacité n'est en aucune façon suffisante pour faire passer les quantités prévues d'électricité. Étant donné les avantages de l'itinéraire terrestre, il semble également intéressant de planifier un tracé passant par l'Algérie ou la Tunisie, puis la Sardaigne et la Corse vers l'Italie, ou alors de choisir la voie de la Tunisie ver la Sicile et l'Italie du Nord.

\section{d. Les pentes critiques}

Dans une autre étape, il est nécessaire d'intégrer dans l'analyse SIG l'énergie du relief car les voies de passage des lignes électriques doivent éviter les reliefs abrupts. On ne peut pas construire des pylônes sur des pentes de plus de $100 \%\left(45^{\circ}\right)$ car c'est techniquement impossible ou trop coûteux. Le mieux est de faire passer les lignes électriques le long des vallées qui structurent les grandes unités de relief. Pour déterminer les zones d'exclusion on utilise les données de la mission SRTM (NASA 2005). 


\section{Résultats : détermination du tracé des lignes haute tension}

16 Ce qu'on peut retenir dans l'ensemble, c'est que la construction des lignes électriques est moins coûteuse en terrain plat inculte. À l'inverse, elle est d'autant plus coûteuse qu'elle doit franchir de nombreuses pentes fortes et que les surfaces maritimes, agricoles et forestières occupent une place importante. À cela s'ajoutent des facteurs coûts spécifiques à chaque pays (par exemple les coûts du travail) qui sont représentés dans un calque particulier (fig. 2). Ainsi, chaque pixel contient les informations suivantes: voie terrestre ou maritime, inclinaison des pentes, type d'utilisation du sol et fond socio-économique. Il est important de souligner que l'analyse ne contient pas les coûts proprement dits, mais les différents facteurs coûts qui varient en fonction des conditions. Par exemple pour une voie terrestre, elle est affectée du coefficient 0,6 et de 2 pour un câble sous-marin. Pour les différentes pentes les facteurs coûts augmentent avec l'inclinaison (coefficients de 1 à 1,5 ).

Figure 2

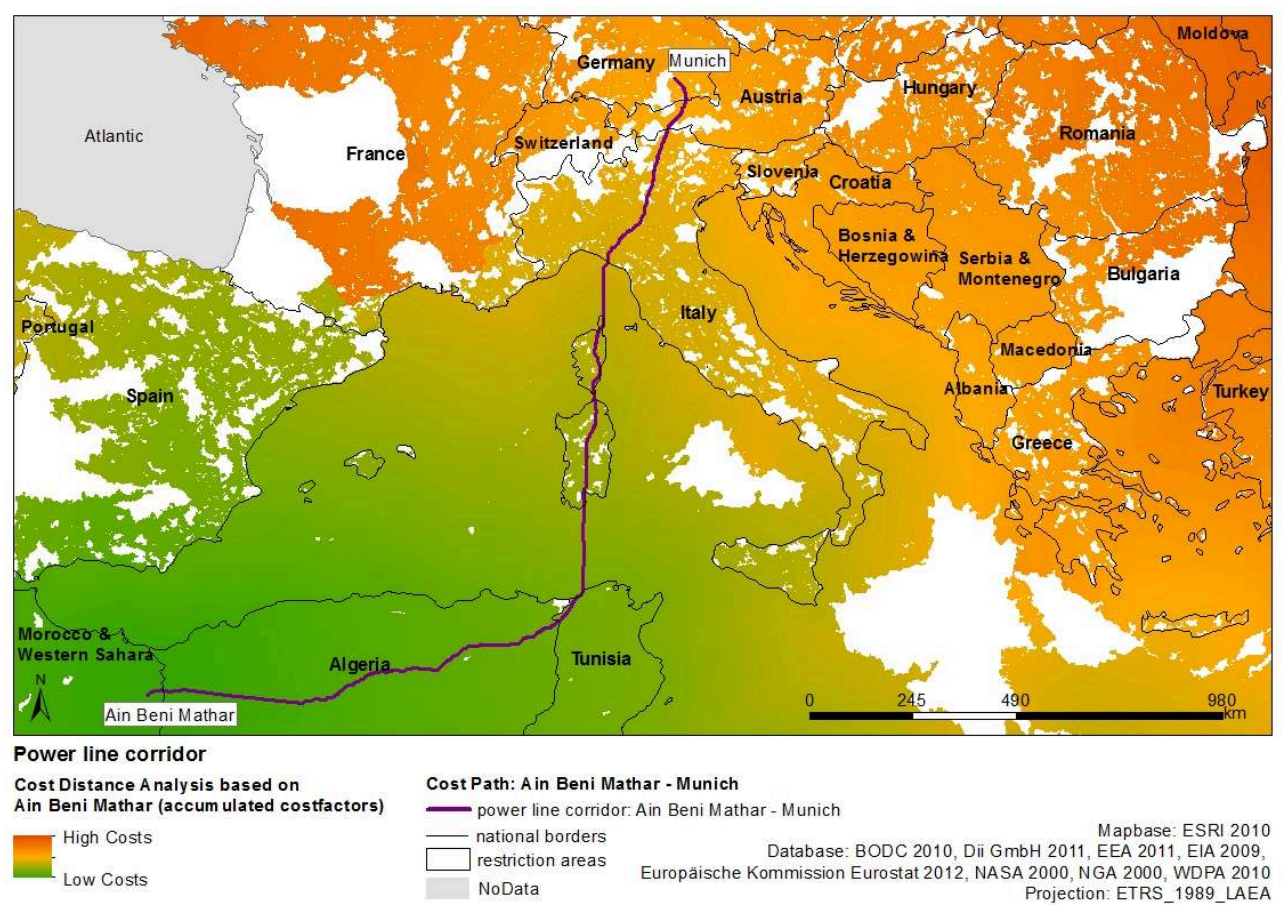

17 Grâce au SIG, on peut par la méthode d'analyse des distances-coûts à l'aide des masques d'exclusion (fig. 1), déterminer les tracés de lignes les plus avantageux. Cela est illustré à titre d'exemple pour le tracé entre le Maroc et l'Allemagne (fig. 2): le point de départ de la ligne est la centrale hybride marocaine à Ain Beni Mathar. On a choisi Munich comme point d'arrivée. Le tracé de ligne optimal traverse d'abord les pays situés à l'est, l'Algérie et la Tunisie. La traversée de la Méditerranée est facilitée par le passage à travers la Sardaigne et la Corse, puis de là à travers les Alpes par l'Italie du Nord et l'ouest de l'Autriche jusqu'à la capitale bavaroise. 


\section{Conclusion} concerter et faire preuve de leur capacité au compromis. Les intérêts nationaux constituent un obstacle sur la voie d'un système énergétique transcontinental. L'introduction d'un tarif d'achat transnational dont en tout cas tous les États nationaux ne profiteraient pas, serait fondamentale pour le succès de Desertec (Schmitt 2012, p. 256). Il faudrait cependant faire attention à ce que tous les États nationaux partenaires puissent tirer profit du projet. Le tarif d'achat pourrait profiter non seulement aux pays producteurs, mais aussi aux pays de transit. Dans ces conditions, l'Algérie pourrait alors servir de pays de transit pour du courant produit au Maroc.

Pour déterminer d'autres voies de passage des lignes électriques, il faut noter que les informations sur les sites d'implantation des centrales en Afrique du Nord n'existent que pour un cas de figure. Il est alors envisageable de déterminer les tracés à partir des centres de consommation européens en direction des lieux de production. On peut alors avoir une idée pour savoir où il serait le plus intéressant d'installer une centrale solaire-thermique ou photovoltaïque. L'analyse SIG peut ainsi être utilisée comme méthode pour identifier les points de départ les plus adaptés pour les lignes électriques. En tout cas, dans le cadre de cette étude, on a réussi à structurer le vaste espace d'étude et à avoir un regard sur les dimensions spatiales du transport intercontinental du courant électrique. On a pu déterminer plusieurs corridors qui conviendraient à un futur tracé, mais qui demandent encore à être affinés localement.

\section{BIBLIOGRAPHY}

ABB AB, 2008, Submarine Cable Link, The NorNed HVDC Connection, Norway - Netherlands, http://www05.abb.com/global/scot/scot245.nsf/veritydisplay/

2402665447f2d054c12571fb00333968/\$file/

project\%20norned\%20450\%20kv\%20dc\%20mi\%20sub.pdf, 05/12/2013.

BINE Informationsdienst, 2011, Solarthermische Kraftwerke: Deutsch-Marokkanische Zusammenarbeit, http://www.bine.info/hauptnavigation/publikationen/news/news/ solarthermische-kraftwerke-deutsch-marokkanische-zusammenarbeit/?artikel=2120, $06 / 11 / 2012$.

Bosch S., 2010, „Sauberer Strom aus der Wüste - Der Ausbau von konzentrierenden solarthermischen Systemen zur Sicherung der Energie- und Wasserversorgung in der EUMENARegion“, in Friedmann A., Hilpert M., Jacobeit J., Kunstmann H., Peyke G., Reller A., Schneider T., Thieme K., Timpf S., Wetzel K.-F. \& Wieczorek U. (dir.), Geographica Augustana, Bd. 8, Augsburg.

British Oceanographic Data Centre (BODC), 2010, Gridded bathymetry Datasets, https:// www.bodc.ac.uk/data/online_delivery/gebco/,06/12/2013.

Dannenberg M., Duracak A., Hafner M. \& Kitzing S., 2012, Energien der Zukunft - Sonne, Wind, Wasser, Biomasse, Geothermie, Darmstadt.

Revue Géographique de l'Est, vol. 55 / n¹-2 | 2015 
Desertec Foundation, 2009, Red Paper Das Desertec Konzept im Überblick, www.desertec.org/ downloads/summary_de.pdf, 05/12/2013.

Deutsches Zentrum für Luft- und Raumfahrt (DLR), 2006, Trans-Mediterranean Interconnection for Concentrating Solar Power, http://www.dlr.de/tt/Portaldata/41/Resources/dokumente/ institut/system/projects/TRANS-CSP_Full_Report_Final.pdf, 05/12/2013.

Deutsches Zentrum für Luft- und Raumfahrt (DLR), 2009a, Concentrating Solar Power for the Mediterranean Region, http://www.dlr.de/tt/Portaldata/41/Resources/dokumente/institut/ system/projects/MED-CSP_Full_report_final.pdf, 05/12/2013.

Deutsches Zentrum für Luft- und Raumfahrt (DLR), 2009b, Characterisation of Solar Electricity Import Corridors from MENA to Europe, http://www.dlr.de/tt/Portaldata/41/Resources/ dokumente/institut/system/publications/Solar_import_DLR_2009_07.pdf, 05/12/2013.

Deutsches Zentrum für Luft- und Raumfahrt (DLR), 2012, Corine Landcover -

Bodenbedeckungsdaten für Deutschland, http://www.corine.dfd.dlr.de/intro_de.html, 05/12/2013.

Dii GmbH, 2011, Input Transmission Data to WG generation, München.

Dii GmbH, 2012, Desert Power 2050, http://www.dii-eumena.com/fileadmin/flippingbooks/ dp2050_exec_sum_dt_web.pdf, 05/12/2013.

ESRI, 2010, Data \& Maps, http://www.arcgis.com/home/group.html? owner=esri\&title=ESRI\%20Data\%20\%26\%20Maps\&content=all, 06/12/2013.

Europa, 2008, Zusammenfassungen der EU-Gesetzgebung, http://europa.eu/ legislation_summaries/regional_policy/management/g24218_de.htm, 05/12/2013.

Europäische Kommission Eurostat, 2012, Suche Datenbank, http://epp.eurostat.ec.europa.eu/ portal/page/portal/statistics/search_database, 06/12/2013.

Europäische Union (EU), 2003, Verordnung (EG) Nr. 1059/2003 des Europäischen Parlaments und des Rates vom 26. Mai 2003 über die Schaffung einer gemeinsamen Klassifikation der Gebietseinheiten für die Statistik (NUTS), http://eur-lex.europa.eu/LexUriServ/LexUriServ.do? uri=OJ:L:2003:154:0001:0041:DE:PDF, 05/12/2013.

European Environment Agency (EEA), 2011a, Natura 2000 data - the European network of protected sites, http://www.eea.europa.eu/, 05/12/2013.

European Environment Agency (EEA), 2011b, Corine Land Cover 2000 raster data - version 15 (08/2011), http://www.eea.europa.eu/data-and-maps/data/corine-land-cover-2000-raster-1, $06 / 12 / 2013$.

Galsi, 2011, Gasdotto Algeria Italia via Sardegna, Milano, http://www.galsi.com/, 05/12/2013.

Heuer S., 2011, „Strom aus der Schüssel“, in Technology Review - Das MIT Magazin für Innovation, 1, Spezialheft Energie, p.54-61.

Heup J., 2012, Spiegel mit Kratzern, in Neue Energie - Das Magazin für Erneuerbare Energien, 9, p.83-86.

International Union for Conservation of Nature (IUCN), 2012, IUCN Protected Area Management Categories, http://www.iucn.org/about/work/programmes/gpap_home/gpap_quality/ gpap_pacategories/, 05/12/2013.

Kaltschmitt M., Streicher W., Wiese A. (dir.), 2006, „Erneuerbare Energien - Systemtechnik“, Wirtschaftlichkeit, Umweltaspekte, 4. Aufl., Berlin. 
Lessner A., 2010, „CSP vor neuem Schub“, in Erneuerbare Energien - Das unabhängige Monatsmagazin für die Zukunftsenergien, 2, p.52-55.

Leuthold F., Weigt H. \& v. Hirschhausen C., 2008, ELMOD - A Model of the European Electricity Market, http://papers.ssrn.com/sol3/Delivery.cfm/SSRN_ID1169082_code857256.pdf? abstractid $=1169082 \&$ mirid $=1,05 / 12 / 2013$.

Löfken J.-O., 2011a, „Vorreiter Afrika“, in Technology Review - Das MIT Magazin für Innovation, 1, Spezialheft Energie, p.62-63.

Löfken J.-O., 2011b, „Afrika entdeckt die Sonne“, in Technology Review - Das MIT Magazin für Innovation, 6, p.26-32.

National Aeronautics and Space Administration (NASA), 2005, Shuttle Radar Topography Mission, http://www2.jpl.nasa.gov/srtm/dataprod.htm, 05/12/2013.

National Aeronautics and Space Administration (NASA), 2009, Jet Propulsion Laboratory, California Institute of Technology, http://www2.jpl.nasa.gov/srtm/index.html, 06/12/2013.

National Geospatial Intelligence Agency, 2009, https://www.nga.mil/ProductsServices/Pages/ default.aspx, 06/12/2013.

Quaschning V., 2011, Regenerative Energiesysteme - Technologie, Berechnung, Simulation, München.

Rentzing S., 2011, „Im Schatten der Photovoltaik“, in Neue Energie - Das Magazin für Erneuerbare Energien, 10, p.48-51.

Schmitt T., 2012, „Postfordistische Energiepolitiken? Das Desertec-Konzept als Szenario zur Restrukturierung der Energieversorgung in der EUMENA-Region“, Zeitschrift für Wirtschaftsgeographie, 4, p.244-263.

Schwarzburger H., 2012a, „Gebündelte Energie“, in Erneuerbare Energien - Das Magazin, 5, p. 62-65.

Schwarzburger H., 2012b, „Voll konzentriert“, in Erneuerbare Energien - Das Magazin, 11, p. 56-59.

Trieb F., 2012, „Strom aus der Wüste - DLR-Studien zum Projekt Desertec“, in Bührke T. \& Wengenmayr R. (dir), Erneuerbare Energien - Konzepte für die Energiewende, Weinheim. Tunur, 2012, Submarine Electricity Link, http://www.tunur.tn/index.php/en/submarineelectricity-link.html, 05/12/2013.

US Energy Information Administration (EIA), 2009, http://www.eia.gov/cfapps/ipdbproject/ IEDIndex3.cfm?tid=2\&pid=2\&aid=2, 05/12/2013.

US Geological Survey (USGS), 2000, Shuttle Radar Topography Mission (SRTM) -“Finished", http://eros.usgs.gov/\#/Find_Data/Products_and_Data_Available/SRTM, 24/08/2012.

Wandler R., 2011, „Der Sonnenkönig“, in Neue Energie - Das Magazin für Erneuerbare Energien, 7, p.92-95.

World Database on Protected Areas (WDPA), 2010, http://www.wdpa.org/, 06/12/2013.

WWF Deutschland, 2008, Hintergrundinformation Internationale Schutzgebiets-Kategorien der IUCN, http://www.wwf.de/fileadmin/fm-wwf/Publikationen-PDF/

IUCN_Schutzgebietskriterien.pdf, 05/12/2013.

Zimmermann J.-R. \& Weinhold N., 2011, „Das Netz wächst zusammen“, in Neue Energie - Das Magazin für Erneuerbare Energien, 12, p.17-23. 


\section{ABSTRACTS}

An oversupply of solar radiation in North Africa as well as a high energy consumption in the economic centers of Central Europe give rise to cross-link the renewable energy supply intercontinental. Fundamental to this is the development of high-performance power line routes. This alone can increase the energy of the places of production economically transported to the distant consumption centers. However, now there is little information about how an optimal power line route is to be determined. In the present study, therefore, a methodology is presented, which makes it possible to optimize power transfer between North Africa and Europe in space. To this end, the decisive spatial factors were detected in the planning of power line routes and displayed using GIS. By way of example, the optimal course of a current power line between the Moroccan Ain Beni Mathar power plant site and consumption center München is calculated at the end of the study.

Un surplus d'ensoleillement en Afrique du Nord, ainsi qu'une forte consommation d'énergie dans les centres économiques d'Europe centrale donnent l'occasion de réaliser un réseau intercontinental pour les énergies renouvelables. Pour cela, il est nécessaire de construire des lignes à très haute tension de transport d'électricité. C'est le seul moyen de transporter le courant des centres de production aux centres de consommation de manière économiquement rentable. Jusqu'à présent on a peu de connaissances sur la manière de déterminer le tracé optimal de ces lignes. Dans le cadre de cette étude, on présente une méthode qui permet d'optimiser le transport du courant entre l'Afrique du Nord et l'Europe. Grâce à l'utilisation de SIG on a déterminé et représenté les facteurs importants ayant une influence spatiale pour le tracé des lignes. À titre d'exemple, on présente à la fin de l'étude le tracé optimal d'une ligne électrique entre la centrale marocaine de Ain Beni Mathar et le centre de consommation de Munich.

Ein Überangebot an solarer Einstrahlung im Norden Afrikas sowie ein hoher Energieverbrauch in den Wirtschaftszentren Mitteleuropas geben Anlass dazu, die regenerative Energieversorgung interkontinental $\mathrm{zu}$ vernetzen. Grundlegend hierfür ist der Ausbau leistungsstarker Stromleitungstrassen. Allein dadurch lässt sich die Energie von den Orten der Produktion zu den weit entfernten Verbrauchszentren wirtschaftlich transportieren. Bislang liegen jedoch wenige Erkenntnisse darüber vor, wie ein optimaler Trassenverlauf zu ermitteln ist. Im Rahmen der vorliegenden Studie wird deshalb eine Methodik vorgestellt, die es ermöglicht, den Stromtransport zwischen Nordafrika und Europa räumlich zu optimieren. Hierzu wurden die entscheidenden räumlichen Einflussfaktoren bei der Planung von Stromleitungstrassen erfasst und mittels GIS dargestellt. Beispielhaft wird am Ende der Studie der optimale Verlauf einer Stromtrasse zwischen dem marokkanischen Kraftwerksstandort Ain Beni Mathar und dem Verbrauchszentrum München berechnet.

\section{INDEX}

Schlüsselwörter: Desertec, Energietransport, erneuerbare Energien, Gleichstromtrasse, interkontinentales Energiekonzept, Solarenergie, Szenario

Keywords: DC route, Desertec, energy transport, intercontinental energy concept, renewable energy, scenario, solar energy

Mots-clés: concept énergétique intercontinental, Desertec, énergie solaire, énergies renouvelables, scénario, tracé de ligne à courant continu, transport d'énergie 


\section{AUTHORS}

\section{KATRIN WALTER}

Universität Augsburg - Katrin.walter@geo.uni-augsburg.de

\section{STEPHAN BOSCH}

Universität Augsburg - Stephan.bosch@geo.uni-augsburg.de 